\title{
Application of the Laplace transform to non-rectilinear momentary problems
}

\author{
Koram Samuel Sakyi ${ }^{1 *} \quad$ Jian-Fei $\mathrm{Lu}^{2}$ \\ 1. School of Civil Engineering and Mechanics, Jiangsu University, PO box 321, Zhenjiang, China \\ 2. School of Civil Engineering and Mechanics, Jiangsu University, PO box 321, Zhenjiang, China
}

\begin{abstract}
.
The cross presentation of the Laplace transform method and the finite variance method to one-dimensional nonaligned momentary heat conduction problems is considered. The calculation is discretized in the interplanetary area by the finite difference method. The nodal heats are at that point changed by the use of the Laplace transform method. The changed heats are reversed mathematically to get the outcomes in the corporeal measures. This report depicts that the current technique does not need to achieve a time-pacing method. Therefore the outcomes at whatever time can be calculated in the time area deprived of any sequential calculations. The current outcomes are likened in tables with those achieved by the straight variational technique and other means. However, it is observed that their outcomes are in right agreement with per capita other. It can be established that the current cross technique is consistent and efficient. Furthermore, two diverse linearization methods for the nonaligned peripheral state are examined.
\end{abstract}

Keywords: Laplace transform, finite change method, non-rectilinear momentary problems

DOI: $10.7176 /$ ISDE/10-1-04

\subsection{Introduction}

In the arithmetic means the finite element method (FEM) and the finite variance method are often used. In earlier arithmetical approaches, nonaligned momentary or transitory heat transmission problems were resolved mostly by the Crank-Nicolson technique or the time amalgamation technique. The time amalgamation technique with the median rule has been verified to maintain its total stability in the nonaligned system. It is essential to use lesser time paces to evade undesirably arithmetical alternations in the result when the Crank-Nicolson technique is used. It can be set up from Orivuori's work that the deviation of the results obtained by means of the Crank-Nicolson technique is actually simple for minor values of time. The supplementary demerit in the use of these two approaches to momentary or transitory problems is that reiteration is required at every time pace. This method will have a tendency to upsurge the rate once only lengthy time results are needed. To decrease these hindrances, the current technique is examined. Several instances are examined to exemplify the arithmetical precision of the cross technique. It is however observed that the current outcomes are in right agreement with those gotten by the use of the straight variational technique and other approaches. Moreover, the outcomes are even over the whole area.

\subsection{Analysis}

Taking a look at the dimensionless procedure of the leading (or governing) differential equation for one-dimensional momentary heat conduction in a slab by temperature-dependent current conductivity can be articulated as:

$$
\frac{\partial}{\partial x}\left(k(T) \frac{\partial T}{\partial x}\right)=\frac{\partial T}{\partial t}
$$

for $0<\mathrm{x}<1, \mathrm{t}>0$

with peripheral settings presented in the subsequent formulae:

$$
\begin{aligned}
& \mathrm{k}(\mathrm{T})-+\mathrm{qo}(\mathrm{t})-\operatorname{Bio}\left(\mathrm{T}-T_{0 x}\right) \mathrm{ax} \text { at } \mathrm{x}-\mathbf{a} \\
& \mathrm{k}(\mathrm{T})-+\mathrm{ql}(\mathrm{t})+\operatorname{Bil}(\mathrm{T} ' \mathrm{~T} 100)-0 \text { дx }
\end{aligned}
$$

and the preliminary form

$$
T(x, 0)=T_{\text {in }}
$$

where $\mathrm{T}$ in is the preliminary heat, Toc is the heat of the environs, and Bio and Bil are Biot statistics. They are welldefined as Bio hob/k() and Bil- transform: 
Lined difference of current conductivity with heat is expected to be

$$
k(T)=1+\beta T
$$

The discretized method of equation (1), by means of the principal differential procedure, can be articulated as

$$
\begin{aligned}
\frac{\partial T_{i}}{\partial t} & =\frac{1}{l}\left\{\left[(1+\beta T) \frac{\partial T}{\partial x}\right]_{i+1 / 2}-\left[(1+\beta T) \frac{\partial T}{\partial x}\right]_{i-1 / 2}\right\} \\
& =\frac{1}{l}\left[\left(1+\beta \frac{T_{i}+T_{i+1}}{2}\right) \frac{T_{i+1}-T_{i}}{l}-\left(1+\beta \frac{T_{i}+T_{i-1}}{2}\right) \frac{T_{i}-T_{i-1}}{l}\right] \\
& =\frac{1}{l^{2}}\left[T_{i-1}-2 T_{i}+T_{i+1}\right]+\frac{\beta}{2 l^{2}}\left[T_{i}^{2},-2 T_{i}^{2}+T_{i+1}^{2}\right] \quad i=1,2,3, \ldots, n
\end{aligned}
$$

where fabricated nodal points $\mathrm{i}=0$ and $i=\mathrm{n}+\mathrm{I}$ at the exterior of the area are proved in Figure $1 ; \mathrm{I}$ signifies the space amid two nodes.

Let $f(u, v)$ be a countless times differentiable functional equations of $u$ and $v$. At that point its Taylor's series expansion represented as:

$$
f(u, v)=f(\bar{u}, \bar{v})+\left(\frac{\partial f}{\partial u}\right)_{u, v}(u-\bar{u})+\left(\frac{\partial f}{\partial v}\right)_{u, v}(v-\bar{v})+\cdots
$$

Linearization of the nonaligned time Ti Ti by the Taylor's series calculation presents:

$$
T_{i} T_{j}=-\bar{T}_{i} \bar{T}_{j}+\bar{T}_{i} \bar{T}_{j}+\bar{T}_{j} \bar{T}_{i}
$$

Hence, the linearized procedure of equation (4) with equation (6) is

$$
\frac{1}{l^{2}}\left[T_{i-1}-2 T_{i}+T_{i+1}\right]+\frac{\beta}{2 l^{2}}\left[-\bar{T}_{\bar{i}-1}^{2}+2 \bar{T}_{i-1} T_{i}+2 \bar{T}_{i}^{2}-4 \bar{T}_{i} T_{i}-\bar{T}_{\bar{i}+1}^{2}+2 \bar{T}_{i+1} T_{i+1}\right]=\frac{\partial T_{i}}{\partial t}
$$

$$
i=1,2, \ldots, n
$$

where the overbar signifies the earlier repeated result.

Meanwhile, the Laplace transform of an existent equation and its transposed formulas are well-defined as:

$$
\begin{aligned}
& \hat{\phi}(s)=\mathscr{L}[\phi(t)]=\mathrm{e}^{3 \mathrm{t}} 4(\mathrm{t}) \mathrm{dt} \\
& \phi(t)=\mathscr{L}^{-1}[\hat{\phi}(s)]=\frac{1}{2 \pi i} \int_{i-i x}^{i+i x} e^{\prime \prime} \hat{\phi}(s) \mathrm{ds}
\end{aligned}
$$

where $\mathrm{s} \quad \mathrm{v}+\mathrm{iw}(\mathrm{v}, \mathrm{w}$ e R) is a multifaceted numeral.

Let's take the Laplace transform of equation (7) with regards to time areas

$$
\frac{1}{l^{2}}\left(1+\beta \bar{T}_{i-1}\right) \hat{T}_{i-1}-\frac{1}{l^{2}}\left(2+2 \beta \bar{T}_{i}\right) \hat{T}_{i}+\frac{1}{l^{2}}\left(1+\beta \bar{T}_{i+1}\right) \hat{T}_{i+1}+\frac{\beta}{2 s l^{2}}\left(-\bar{T}_{i-1}^{2}+2 \bar{T}_{i}^{2}-\bar{T}_{i+1}^{2}\right)=s \hat{T}_{i}-T_{\text {in }}
$$

Equation (9) can be reorganized as

$$
\left(1+\beta \bar{T}_{i-1}\right) \hat{T}_{i-1}-\left(2+2 \beta \grave{i}+\quad+\left(1+\beta \ddot{i i}+{ }^{1}\right) \hat{T}_{i+1}=\frac{\beta}{2 s}\left(\bar{T}_{\bar{i}-1}^{2}-2 \bar{T}_{i}^{2}+\bar{T}_{i+1}^{2}\right)-T_{\text {in }} l^{2}\right.
$$

The peripheral settings (2) are the nonaligned method. Hence they are of a necessity to be linearized in the current study. At present, two diverse linearization methods will be carefully chosen.

The initial linearization method of the peripheral settings (2) can be specified as

$$
\left(1+\beta \frac{T_{2}+T_{0}}{2}\right) \frac{T_{2}-T_{0}}{2 l}+q_{0}(t)-\mathrm{Bi}_{0}\left(T_{1}-T_{0 \infty}\right)=0
$$




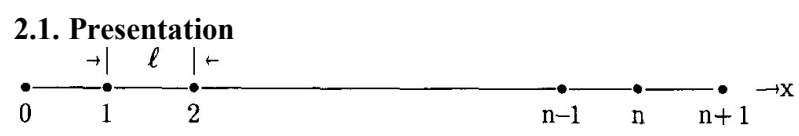

Figure 1. Representation illustration of one-dimensional problems

and

$$
\left(1+\beta \frac{T_{n+1}+T_{n-1}}{2}\right) \frac{T_{n+1}-T_{n-1}}{2 l}+q_{1}(t)+\mathrm{Bi}_{1}\left(T_{n}-T_{1 \propto}\right)=0
$$

The linearization method of equations (1 1) by means of the Taylor's series calculation, equation (6), is

$$
\begin{aligned}
& -\left(1+\quad-+(1+\beta \mathrm{T} 2) \mathrm{T} 2=\frac{\kappa}{n}\left(\bar{T}_{2}^{2}-\bar{T}_{0}^{2}\right)-2 l q_{0}(t)-2 / \mathrm{Bi}_{0} T_{1 \times x}\right. \\
& \quad-\left(1+\beta \bar{T}_{n-1}\right) T_{n-1}+2 / \mathrm{Bi}_{1} T_{n}+\left(1+\beta \bar{T}_{n+1}\right) T_{n+1}=\frac{\beta}{2}\left(\bar{T}_{n+1}^{2}-\bar{T}_{n-1}^{2}\right)-21 \mathrm{qI}(\mathrm{t})+21 \mathrm{BiI} T_{1 \times}
\end{aligned}
$$

Let's take the Laplace transform of equations ( 12) with regards to time areas

$$
-\left(1+\beta \bar{T}_{0}\right) \hat{T}_{0}-2 / \mathrm{Bi}_{0} \hat{T}_{1}+\left(1+\beta \bar{T}_{2}\right) \hat{T}_{2}=\frac{\beta}{2 s}\left(\bar{T}_{2}^{2}-\bar{T}_{0}^{2}\right)-2 l \hat{q}_{0}-\frac{2 / \mathrm{Bi}_{0} T_{0 x}}{s}
$$

and

$$
-\left(1+\beta \bar{T}_{n-1}\right) \hat{T}_{n-1}+2 / \mathrm{Bi}_{1} \hat{T}_{n}+\left(1+\beta \bar{T}_{n+1}\right) \hat{T}_{n+1}=\frac{\beta}{2 s}\left(\bar{T}_{n+1}^{2}-\bar{T}_{n}^{2} 1\right)-2 l \hat{q}_{1}+\frac{2 l \mathrm{Bi}_{1} T_{1 \times}}{s}
$$

The subsequent linearization method of the peripheral settings (2), by means of the principal difference procedure,

$$
\begin{aligned}
& \left(1+\beta T_{1}\right) \frac{T_{2}-T_{0}}{2 l}+q_{0}(t)-\mathrm{Bi}_{0}\left(T_{1}-T_{(1)}\right)=0 \\
& \left(1+\beta T_{n}\right) \frac{T_{n+1}-T_{n-1}}{2 l}+q_{1}(t)+\mathrm{Bi}_{1}\left(T_{n}-T_{1 x}\right)=0
\end{aligned}
$$

provides

Therefore making equations (14) linear using equation (6) can be gotten by the subsequent

$$
\text { linearization methods as }\left(-\mathrm{I}-+\left(\beta \mathrm{T} 2-\beta \mathrm{ï}()-+(1+\beta \mathrm{II}) \mathrm{T} 2=-\bar{T}_{1} \bar{T}_{0}\right)-2 / q_{0}(t)-2 / \mathrm{Bi}_{0} T_{0 \times}\right.
$$

$$
\left(-1-\beta \bar{T}_{n}\right) T_{n-1}+\left(\beta \bar{T}_{n+1}-\beta \mathrm{Tn}_{-} \mathrm{I}+21 \mathrm{BiI}\right) \mathrm{Tn}+(1+\beta \ddot{\mathrm{in}}) \mathrm{Tn}_{+1}
$$

$$
=\beta\left(\bar{T}_{n} \bar{T}_{n+1}-\bar{T}_{n} \bar{T}_{n} \quad 1\right)-21 \mathrm{qI}(\mathrm{t})+21 \mathrm{BiI} T_{1 \times} \quad(1
$$

Let's then take the Laplace transform of the peripheral settings (15) with regards to time is given as:

$$
\begin{aligned}
& \left(-1-+\left(\beta \mathrm{T} 2-\beta \mathrm{i}()-+(1+\beta \mathrm{TI}) \hat{\mathrm{I} 2}=\frac{\beta\left(\bar{T}_{1} \bar{T}_{2}-\bar{T}_{1} \bar{T}_{0}\right)}{s}-2 l \hat{q}_{0}-\frac{2 / \mathrm{Bi}_{0} T_{0 x}}{s}\right.\right.
\end{aligned}
$$

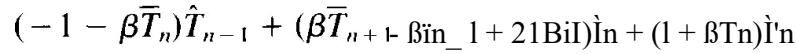

$$
\begin{aligned}
& +1 \\
& =\frac{\beta\left(\bar{T}_{n} \bar{T}_{n+1}-\bar{T}_{n} \bar{T}_{n-1}\right)}{s}-2 / \hat{q}_{1}+\frac{2 / \mathrm{Bi} T_{1} T_{1 x}}{s}
\end{aligned}
$$

The change of equation (9) and the peripheral settings (13) or (16) can produce the subsequent vector matrix equation:

$$
[k]\{\hat{T}\}=\{f\}
$$

where $[\mathrm{k}]$ is an $(\mathrm{n} \times \mathrm{n})$ crew matrix with multifaceted numeral, $\{\mathrm{T}\}$ is an $(\mathrm{n} \times 1)$ vector signifying the indefinite changed heats, and $\{f\}$ is an ( $\mathrm{n} \times 1)$ vector signifying the compelling terms.

A preliminary result $\{\mathrm{T}\}$ is predicted to calculate $[\mathrm{k}]$ and $\{\mathrm{f}\}$, and also the use of the direct Gaussian elimination procedure and the arithmetical transpose of Laplace transform to equation (17) can produce the nodal heats in the corporal measures. The Laplace transform computational method is accomplished frequently till the comparative blunders among the present nodal heats and the standards at the preceding reiteration are all not more than a value of easiness. 
$\frac{\left|T_{i}^{(m)}-T_{i}^{(m+1)}\right|}{T_{i}^{(m)}} \longrightarrow 0$

$$
\text { as } m=0,1,2, \ldots, \text { i } \quad 1,2, \ldots, n
$$

where the superscript $\mathrm{m}$ signifies the mth reiteration. Though, the merging standard presented in equation ( 18) does not at all times pledge the precision of the arithmetical result for iterative systems. The statistic is well-known to all. To examine the accurateness of the cross system, several descriptive samples will be examined.

\subsection{Numerical samples/ results.}

\subsection{First Sample (specified by Krajewsk'i ${ }^{7}$ )}

Contemplate on a momentary heat transmission problem in a one-dimensional slab specified by Krajewski ${ }^{7}$ and Mehta. ${ }^{8}$ Its leading (or governing) differential equation, peripheral state

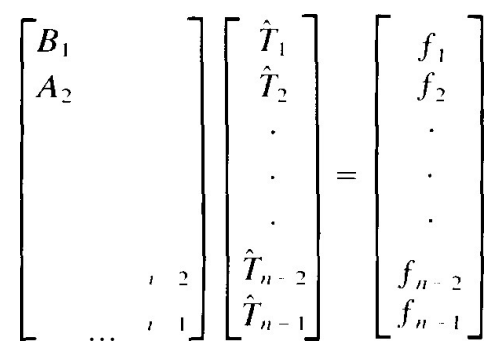

where

$$
\begin{aligned}
& A_{i}=1+\beta \bar{T}_{i-1} \quad i=2,3, \ldots, n-1 \\
& B_{i}=-2-2 \beta \bar{T}_{i}-I^{2} s \quad i=1,2, \ldots, n-1 \\
& C_{i}=1+\beta \bar{T}_{i+1} \quad i=1,2, \ldots, n-1 \\
& f_{1}=\frac{\beta}{\mathrm{s}}\left(\bar{T}_{2}^{2}-\bar{T}_{\overline{1}}^{2}\right) \\
& f_{i}=\frac{\beta}{2 s}\left(\bar{T}_{i-1}^{2}-2 \bar{T}_{i}^{2}+\bar{T}_{i+1}^{2}\right) \\
& \quad i=2,3, \ldots, n-2 \\
& f_{n-1}=\frac{\beta}{2 s}\left(\bar{T}_{n-2}^{2}-2 \bar{T}_{n-1}^{2}+\bar{T}_{n}^{2}\right)-C_{n-1} / s
\end{aligned}
$$

The calculated outcomes gotten as of equation (22) are enumerated in Tables $1-3$ and Figure 2 by means of I I nodes in the interplanetary area. A contrast of the current results with those specified by Krajewski ${ }^{7}$ and Mehta ${ }^{8}$ is elaborated in Tables $1-3$ and Figure 2, correspondingly. However, it can be established from Tables $1-3$ that the outcomes of Krajewski ${ }^{7}$ displayed arithmetical alternations for lesser values of dictions, and preliminary form are articulated as

$$
\frac{\partial}{\partial x}\left[(1+\beta T) \frac{\partial T}{\partial x}\right]=\frac{\partial T}{\partial t}
$$

for $0<\mathrm{x}<1$

$$
\begin{gathered}
\frac{\partial T(0, t)}{\mathrm{ax}}=0 \\
T(1, t)=1 \\
T(x, 0)=0
\end{gathered}
$$

The discretized method of equation (19a), by means of the dominant change procedure, is specified as 
Let's take the Laplace transform of equation (20) with regards to time provides

$$
\frac{\hat{T}_{2}-\hat{T}_{0}}{21}=0
$$

Consequently the resultant scheme of numerical equation for the initial sample can be inscribed in the subsequent matrix formula:

$$
\left[\begin{array}{llll}
B_{1} & & \\
A_{2} & & \\
& & \\
& & & \\
& & & 2 \\
& \ldots & 1 & 1
\end{array}\right]\left[\begin{array}{c}
\hat{T}_{1} \\
\hat{T}_{2} \\
\cdot \\
\cdot \\
\cdot \\
\hat{T}_{n-2} \\
\hat{T}_{n-1}
\end{array}\right]=\left[\begin{array}{c}
f_{1} \\
f_{2} \\
\cdot \\
\cdot \\
\cdot \\
f_{n-2} \\
f_{n-1}
\end{array}\right]
$$

Though, the current technique does not consume this problem. Additionally, Tables $1-3$ likewise display no extraordinary change amid the current results and the outcomes of Krajewski ${ }^{7}$ for $t>0.2$. The succeeding deduction displays the current cross technique.

Table 1. Contrast of several arithmetical outcomes for $\mathrm{t}=0.020 .2$

\begin{tabular}{|c|c|c|c|c|c|}
\hline \multirow[b]{2}{*}{$\begin{array}{l}\mathrm{t} \\
\mathrm{ns}\end{array}$} & \multirow{2}{*}{$\begin{array}{r}2.5 \times 10 \\
6.4 \times 10 \\
2.8 \times 10 \\
1.2 \times 10 \\
4.4 \times 10 \\
\times \\
\text { solutio }\end{array}$} & \multirow{2}{*}{$\begin{array}{l}6 \\
6 \\
5 \\
4 \\
4 \\
4\end{array}$} & & & \multirow[b]{2}{*}{$\begin{array}{c}\text { Second } \\
\text { variational }\end{array}$} \\
\hline & & & Galerkin & variational & \\
\hline \multicolumn{2}{|c|}{0.00 .0630} & & & 0.0633 & 0.06442 \\
\hline \multicolumn{2}{|c|}{0.10 .04481} & & & 0.04482 & 0.04498 \\
\hline \multicolumn{2}{|c|}{0.20 .01440} & & & 0.0138 & 0.0168 \\
\hline \multicolumn{2}{|c|}{0.30 .0228} & & & -0.0235 & -0.0195 \\
\hline 0. & 40.0465 & 0.0475 & 0.0438 & & \\
\hline 0.5 & 0.0150 & 0.0405 & 0.0420 & 0.0416 & \\
\hline 0.6 & 0.0455 & 0.0112 & 0.0095 & 0.0024 & \\
\hline 0.7 & .12150 .125 & & 0.1231 & 0.1047 & \\
\hline 0.8 & 0.2834 & 0.3168 & 0.3152 & 0.2849 & \\
\hline 0.9 & 0.5738 & 0.6032 & 0.6021 & 0.5697 & \\
\hline 1.0 & 1.0000 & & .0000 & 1.0000 & 1.0000 \\
\hline
\end{tabular}

Krajewski7 


\section{2..Submission}

Table 2. Contrast of several arithmetical outcomes for $t=0.2$ and -0.3

\begin{tabular}{|c|c|c|c|c|}
\hline \multirow[b]{2}{*}{$X$} & \multirow[b]{2}{*}{$\begin{array}{c}\text { Present } \\
\text { solutions }\end{array}$} & \multicolumn{3}{|c|}{ Krajewski7 } \\
\hline & & Galerkin & variational & $\begin{array}{l}\text { Second } \\
\text { variational }\end{array}$ \\
\hline 0.0 & 0.1823 & o. 1807 & 0.1805 & 0.1763 \\
\hline 0.1 & 0.1901 & o. 1888 & 0.1886 & 0.1852 \\
\hline 0.2 & 0.2134 & 0.2131 & 0.2128 & \\
\hline 0.3 & 0.2527 & 0.2537 & 0.2532 & 0.2540 \\
\hline 0.4 & 0.3084 & 0.3107 & 0.3100 & 0.3125 \\
\hline 0.5 & 0.3810 & 0.3841 & 0.3833 & 0.3867 \\
\hline 0.6 & 0.4710 & 0.4740 & 0.4732 & 0.4764 \\
\hline 0.7 & 0.5784 & 0.5805 & 0.5796 & 0.5819 \\
\hline 0.8 & 0.7032 & 0.7036 & 0.7029 & 0.7037 \\
\hline 0.9 & 0.8444 & 0.8434 & 0.8430 & 0.8425 \\
\hline 1.0 & 1.0000 & 1.0000 & 1.0000 & I .OOOO \\
\hline
\end{tabular}

Table 3. Contrast of several arithmetical outcomes for $t=1.0$ and $\beta 0.2$

Krajewski7

\begin{tabular}{lcccc}
\cline { 3 - 4 } $\mathrm{x}$ & $\begin{array}{c}\text { Present } \\
\text { solutions }\end{array}$ & Galerkin & variational & $\begin{array}{c}\text { Second } \\
\text { Variational }\end{array}$ \\
\hline & & & & \\
& & & & \\
\hline 0.0 & 0.8329 & 0.8412 & & 0.8480 \\
0.1 & 0.8349 & 0.8432 & 0.8431 & 0.8500 \\
0.2 & 0.8408 & 0.8493 & 0.8492 & 0.8558 \\
0.3 & 0.8507 & 0.8588 & 0.8589 & 0.8651 \\
0.4 & 0.8644 & 0.8720 & 0.8775 \\
0.5 & 0.8809 & 0.8879 & 0.8878 & 0.8928 \\
0.6 & 0.9007 & 0.9064 & 0.9063 & 0.9150 \\
0.7 & 0.9231 & 0.9273 & 0.9272 & 0.9304 \\
0.8 & 0.9474 & 0.9500 & 0.9499 & 0.9522 \\
0.9 & 0.9733 & 0.9744 & 0.9743 & 0.9755 \\
1.0 & 1.0000 & 1.0000 & 1.0000 & 1.0000 \\
\hline
\end{tabular}

is dependable and precise for nonaligned momentary or transitory problems. In overall, four or five reiterations are enough for the current technique to get a convergent heat supply of the first sample at a precise time. Mehta ${ }^{8}$ used the iterative method to control the momentary or transitory heat supply of the first sample. Figure 2 make known the iterative process specified by Mehta $^{8}$ possibly will be deficient in precision.

3.3. Second Sample (specified by Mastanaiah and Muthunayagam ${ }^{9}$ )

Sample 2 examines a similar problem specified by Mastanaiah and Muthunayagam ${ }^{9}$ The leading equation, peripheral settings, and preliminary state of the second sample are specified as

$$
\frac{\partial}{\partial x}\left[(1+\beta T) \frac{\partial T}{\partial x}\right]=\frac{\partial T}{\partial t}
$$

for $0<x<1$

$$
\underline{\partial T}(0, t)=\mathrm{Bi}_{0}[T(0, t)-1]
$$

$$
\frac{\partial T(1, t)}{Ð x}=0
$$

$$
T(x, 0)=0 \quad \text { (24c) }
$$




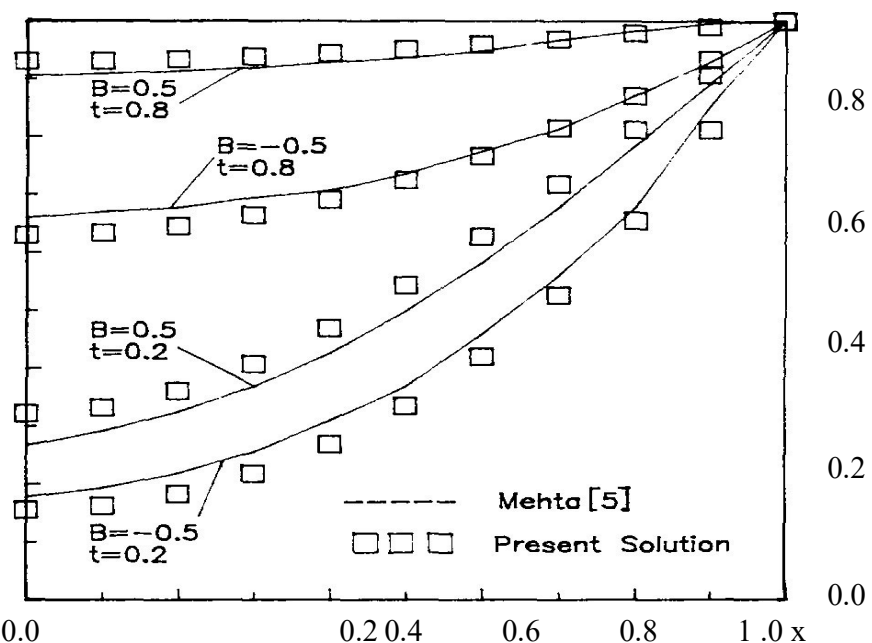

Figure 2. Contrast of the current results with those specified by Mehta ${ }^{8}$

The subsequent results for equations (1) and (24) are itemized in Tables $4-6$. Tables 4 and 5 likewise display a contrast of the current results with the best linearization result specified by Mastanaiah and Muthunayagam ${ }^{9}$ and the finite change result, ${ }^{9}$ correspondingly. The finite change result (FCR) was gotten by means of Crank-Nicolson implied method with two time heights.

However, it is understood that the current results are not merely in right contract with the finite change result, but likewise more precise than the best linearization result (BLR). This deduction additionally proves that the current technique has right precision. Four or five repetitions are enough to get the convergent results for equations of Sample 2 at a precise time.

Consequently the convergent frequency of the current cross technique is qicker. In actual fact, the peripheral form (24a) must not be in a lined method. That is to say, the peripheral form (24a) must be substituted by the subsequent nonaligned procedure. ${ }^{10}$

$$
(1+\beta T) \frac{\partial T}{\partial x}=\mathrm{Bi}_{0}(T-1)
$$

The initial linearization procedure of the peripheral form

(25) gotten as of (12a) is

$$
\begin{array}{r}
-(1+-21 \mathrm{Bi} 0 \mathrm{~T} 1+(1+\beta \mathrm{T} 2) \mathrm{T} 2 \\
=\frac{\beta}{-}\left(\bar{T}_{2}^{2}-\bar{T}_{0}^{2}\right)-21 \mathrm{Bi}()(26) 2
\end{array}
$$

Consequently the Laplace transform of equation (26) with regards to time is

$$
-\left(1+-21 \text { Bi0Ìl }+(1+\beta \mathrm{T} 2) \mathrm{T} 2 \quad\left(T_{2}^{2}-T_{0}^{2}\right)-\frac{-\cdots-\mathrm{v}}{2 \mathrm{~s}}\right.
$$


transform:

Table..4. Contrast of the current results with BLR ${ }^{9}$ when Bio $\quad 1$ and At $=0.1464$

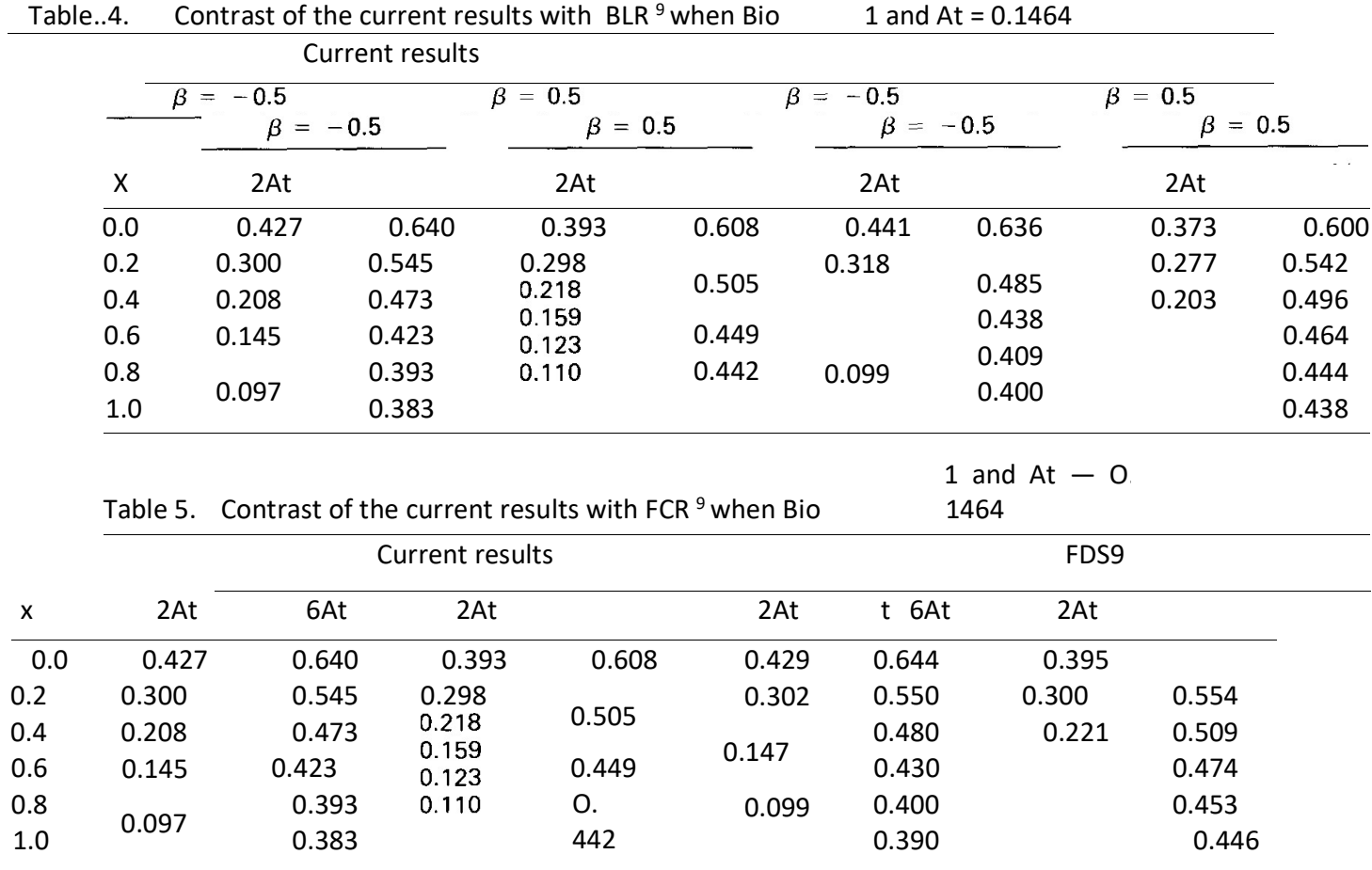

Table 6. Contrast of arithmetical outcomes for two diverse linearizations of the peripheral form when Bio1.0 and At 0.1464

\begin{tabular}{|c|c|c|c|c|c|c|c|c|}
\hline \multirow[b]{3}{*}{$x$} & \multicolumn{4}{|c|}{ Equation (27) } & \multicolumn{4}{|c|}{ Equation (29) } \\
\hline & \multicolumn{2}{|c|}{$\beta=-0.5$} & \multicolumn{2}{|c|}{$\beta=0.5$} & \multicolumn{2}{|c|}{$\beta=-0.5$} & \multicolumn{2}{|c|}{$\beta=0.5$} \\
\hline & $t=2 \Delta t$ & $t=6 \Delta t$ & $t=2 \Delta t$ & $t=6 \Delta t$ & $t=2 \Delta t$ & $t=6 \Delta t$ & $t=2 \Delta t$ & $t=6 \Delta t$ \\
\hline 0.0 & 0.427 & 0.640 & 0.393 & 0.608 & 0.425 & 0.639 & 0.393 & 0.608 \\
\hline 0.2 & 0.300 & 0.545 & 0.298 & 0.551 & 0.299 & 0.544 & 0.298 & 0.552 \\
\hline 0.4 & 0.208 & 0.473 & 0.218 & 0.505 & 0.207 & 0.473 & 0.218 & 0.505 \\
\hline 0.6 & 0.145 & 0.423 & 0.159 & 0.471 & 0.145 & 0.422 & 0.159 & 0.471 \\
\hline 0.8 & 0.109 & 0.393 & 0.123 & 0.449 & 0.109 & 0.392 & 0.123 & 0.449 \\
\hline 1.0 & 0.097 & 0.383 & 0.110 & 0.442 & 0.097 & 0.382 & 0.110 & 0.442 \\
\hline
\end{tabular}

The next linearization procedure of the peripheral form (25) gotten from ( 16a) provides

$$
\begin{aligned}
(-\mathrm{I}-+ & \left(\beta \mathrm{T} 2-\beta \mathrm{T}()-2 / \mathrm{Bi}_{0}\right) \\
& +(1+\beta \mathrm{TI}) \mathrm{T} 2=\beta(\mathrm{TIT} 2-\mathrm{TI} \mathrm{TO})-21 \mathrm{Bi}()(28)
\end{aligned}
$$

Let's take the Laplace transform of the peripheral form (28) with regards to time provides

$$
\left(-1-\beta \bar{T}_{1}\right) \hat{T}_{0}+\left(\beta \bar{T}_{2}-\beta \bar{T}_{0}-2 / \mathrm{Bi}_{0}\right) \hat{T}_{1}
$$

$$
+\left(1+\beta \bar{T}_{1}\right) \hat{T}_{2}=\frac{\beta\left(\bar{T}_{1} \bar{T}_{2}-\bar{T}_{1} \bar{T}_{0}\right)}{\mathrm{s}}-\frac{2 / \mathrm{Bi}_{0}}{}
$$

The outcomes for these two diverse linearization systems of the nonaligned peripheral form at $\mathrm{x}=0$ are presented in Table 6. Table 6 displays no extraordinary change among the outcomes of these two diverse linearization systems.

\subsection{Sample 3}

This sample examines a thick, undeformable block (or slab) whose preliminary heat is equivalent to zero. For $t>0$ the peripheral superficial at $\mathrm{x} 0$ is reserved separately, and the peripheral at $\mathrm{x} I$ is exposed to heat $1-\cos (\mathrm{t})$, which differs with time. The calculated formula of Sample 3 is specified as 


$$
\begin{array}{r}
\frac{\partial}{\partial x}\left[(1+\beta T) \frac{\partial T}{\partial x}\right]=\frac{\partial T}{\partial t} \\
\text { for } 0 \leq x
\end{array}
$$

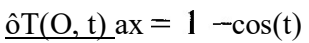

$T(x, 0)=0$

The dimensionless heat supplies of Sample 3 at several time for $\beta=0.3$ and -0.3 are revealed in Table 7. It is thought-provoking to perceive that the connection point of the two curvatures in the direction of $\mathrm{x}$ I with cumulative $t$. For adequately huge $t$, heats for

$\beta=0.3$ are advanced as compared with those for $\beta=-0.3$ all through the block.

\subsection{Presentation}

Table 7. Dimensionless heat supply of Sample 3 at different time for $\beta$

$-0.3$

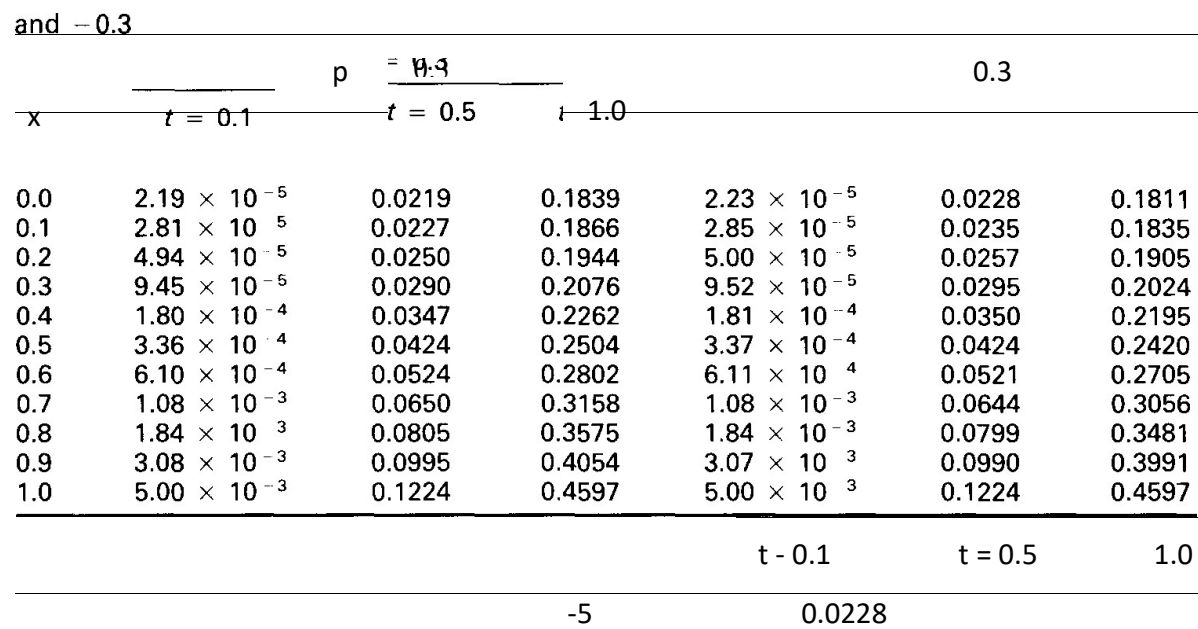

0.1839

0.2262

0.2504

0.2802

0.3158

0.3575

0.4054
O. $194450.02570 .19050 .2076-50.02950 .2024$

$\begin{array}{ccc}4 & 0.0350 & 0.2195 \\ 0.0424 & -4 & \\ 4 & & 0.2420 \\ -3 & 0.0521 & 0.2705 \\ -3 & 0.0644 & 0.3056 \\ 3 & 0.0799 & 0.3481 \\ & 0.0990 & 0.3991\end{array}$

Determining the arithmetical result of a nonaligned momentary heat transmission problem is a complex task. But, the other significant work for nonaligned momentary problems is how to discover a technique that will not be agonize from variabilities in the arithmetical results and can compute the true precise outcome. To display the arithmetical precision of the formula established in the current cross technique, several samples are examined. Furthermore, a problem per the nonaligned peripheral form is likewise well thought-out in the current work. In those problems the linearization by the Taylor's sequence calculation is mostly used to reduce the nonaligned problem into an aligned one.

To the writer's information, the result of $n$ concurrent numerical equations at every time pace is necessary once the Crank-Nicolson procedure is applied. This report suggests that all the interior heats is essential to be calculated at every time pace. This method will have a tendency to upsurge the rate once the results are essential to be carried out over lengthy time phases.

Likewise, it is habitually essential to yield lesser time paces to evade unwanted arithmetical fluctuations in the result. This simple restriction on the time pace possibly will call for an extreme volume of computed time. The benefit of the Laplace transform method is that it can rapidly give a precise result at any definite time. Though, in preceding works the Laplace transform method is used only to resolve lined schemes. No research was conducted on a nonaligned momentary problem for the reason that the Laplace transform method possibly will not effectively do the job. Therefore, it had applied limits. The cross technique has been established to overcome this problem. It is exciting to note that the cross technique can effectively get precise outcomes for nonaligned momentary problems. In overall, four or five reiterations are essential for the current problems. 
Wrobel and Brebbia ${ }^{11}$ suggested that the cross presentation of the peripheral component technique and the Laplace transform method cannot yield right outcomes for problems with time-dependent peripheral settings, due to arithmetical problems in the reverse alteration procedure. This problem does not happen in the current cross technique.

The current technique possibly will be used to solve other problems in two or three dimensions.

\subsection{Terminology}

$\mathrm{BIO}$, Bil Biot numbers $\left(\mathrm{Bio}=h_{0} b / k_{0}, \mathrm{Bi}_{1}=h_{1} b\right.$ b thickness of slab c specific temperature total force vector heat transmission constants at $\mathrm{x} \quad 0$ and

\}

.$h$

$k_{0} \quad$ current conductivity at $\mathrm{t}=0$ current conductivity of block $(1+\beta \mathrm{T})$ total conduction matrix space

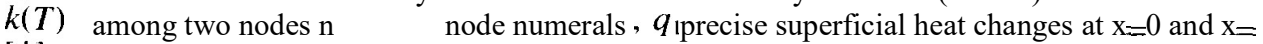

[k] $s \quad$ Laplace transform limit dimensionless heat preliminary temperature ambient heat

$l \quad T$ dimensionless time $\left(\mathrm{aoT} / \mathrm{b}^{2}\right) \mathrm{x}$ dimensionless coordinate $\left(\mathrm{x}^{*} / \mathrm{b}\right)$ *

$T_{\text {in }} \quad$ coordinate

$T_{0 \infty \infty}, T_{1 \times}$

$$
\text { Greek letters orientation thermal diffusivity }\left(k_{0} / \rho_{\beta}\right.
$$

constant (thermal conductivity

coefficient) time compactness

\subsection{Reference}

1. Hughes, T. J. R. Unconditionally stable algorithms for nonlinear heat conduction. Comput. Meth. Appl. Mech. Engrg. 1977, 10, 135-139.

2 Orivuori, S. Efficient method for solution of nonlinear heat conduction problems. Internat. J. Numer. Methods Engrg. 1979, 14. 1461-1476.

3 Shih, T. M. Numerical Heat Transfer. Springer-Verlag, New York, 1984.

4 Dubner, H. and Abate, J Numerical inversion of Laplace transforms by relating them to the finite Fourier cosine transform. J. ACM 1968, 15, 115-123.

5 Durbin. F. Numerical inversion of Laplace transforms: Efficient improvement to Dubner and Abate's method. Comput. J. 1973, 17, 371-376.

6 Honig, G. and Hirdes, U. A method for the numerical inversion of Laplace transforms. J. Comput. Appl. Math. 1984. 9, 113-132.

7 Krajewski, B. On a direct variational method for nonlinear heat transfer. Internat, J. Heat Mass Transfer 1975, $18,495-502$.

8 Mehta, R. C. On the solution of transient conduction with temperature dependent thermal conductivity. J. Heat Transfer 1979, 99, 137-1e39.

9 Mastanaiah, K. and Muthunayagam, A. E. Transient conduction I the finite slab with variable thermal conductivity. A/AA J. 1975, 13, 954-056.

10 Ozisik, M. N. Heat Conduction. John Wiley, New York. 1980. Pp 441-442.

11 Wrobel, I. C. and Brebbia. C. A. A formulation of the boundary element method for axisymmetric transient heat conduction. Internal. J. Heat Mass Transfer 1981, 24, 834-850.

12 Koram, Samuel \& Benjamin, Korankye \& Godson, Kweitsu. (2018). Seismic Response Analysis of Underground Structures. Journal of Environment and Earth Science (IISTE). 